\title{
Genetic Analysis of Human RNA Binding Motif Protein 48 (RBM48) Reveals an Essential Role in U12-Type Intron Splicing
}

Amy E. Siebert ${ }^{a, 1,2}$, Jacob Corll ${ }^{a, 1,3}$, J. Paige Gronevelt ${ }^{a}$, Laurel Levine ${ }^{a}$, Linzi M. Hobbs ${ }^{a}$, Catalina Kenney $^{\mathrm{a}}$, Ruth Davenport ${ }^{\mathrm{b}}$, A. Mark Settles ${ }^{\mathrm{c}, \mathrm{d}, 4}$, W. Brad Barbazuk ${ }^{\mathrm{b}, \mathrm{d}, \mathrm{e}}$, Randal J. Westrick ${ }^{\mathrm{a}, \mathrm{f,g}}$, Gerard J. Madlambayan $^{\mathrm{a}}$ and Shailesh Lal ${ }^{\mathrm{a}, *}$

${ }^{a}$ Departments of Biological Sciences and Bioengineering, Oakland University, Rochester Hills, MI, 48309

${ }^{b}$ Department of Biology, University of Florida, Gainesville, FL, 32611

${ }^{c}$ Horticultural Sciences Department, University of Florida, Gainesville, FL, 32611

${ }^{\mathrm{d}}$ The Genetics Institute, University of Florida, Gainesville, FL, 32611

${ }^{\mathrm{e}}$ The Interdisciplinary Center for Biotechnology Research (ICBR) University of Florida, Gainesville, FL, 32611

${ }^{\mathrm{f}}$ Center for Data Science and Big Data Analytics, Oakland University, Rochester Hills, MI, 48309

${ }^{9}$ Life Sciences Institute, University of Michigan, Ann Arbor, MI, 48109

${ }^{1}$ A.E.S and J.C. contributed equally to this work.

${ }^{2}$ Present address: Versiti Blood Research Institute, Milwaukee, WI, 53226

${ }^{3}$ Present address: Department of Biology, University of Florida, Gainesville, FL, 32611

${ }^{4}$ Present address: Bioengineering Branch, NASA Ames Research Center, M-239, Moffett Field, CA 94043

${ }^{*}$ Corresponding author:

Shailesh Lal

Email: Lal@oakland.edu

Competing Interest Statement: The authors declare no conflict of interest.

Classification: Biological Sciences; Genetics

Keywords: RBM48, RNA processing, cell proliferation, splicing factor, human 


\begin{abstract}
U12-type or minor introns are found in most multicellular eukaryotes and constitute $\sim 0.5 \%$ of all introns in species with a minor spliceosome. Although the biological significance for evolutionary conservation of U12-type introns is debated, mutations disrupting U12 splicing cause developmental defects in both plants and animals. In human hematopoietic stem cells, U12 splicing defects disrupt proper differentiation of myeloid lineages and are associated with myelodysplastic syndrome (MDS), predisposing individuals to acute myeloid leukemia. Mutants in the maize ortholog of RNA Binding Motif Protein48 (RBM48) have aberrant U12-type intron splicing. Human RBM48 was recently purified biochemically as part of the minor spliceosome and shown to recognize the 5' end of the U6atac snRNA. In this report, we use CRISPR/Cas9mediated ablation of RBM48 in human K-562 cells to show the genetic function of RBM48. RNA-seq analysis comparing wild-type and mutant K-562 genotypes found that $42 \%$ of minor intron containing genes (MIGs) have significant U12-type intron retention in RBM48 mutants. Comparing these results to maize rbm48 mutants defined a subset of MIGs disrupted in both species. Mutations in the majority of these orthologous MIGs have been reported to cause developmental defects in both plants and animals. Our results provide genetic evidence that the primary defect of human RBM48 mutants is aberrant U12-type intron splicing, while a comparison of human and maize RNA-seq data identifies candidate genes likely to mediate mutant phenotypes of U12-type splicing defects.
\end{abstract}

\title{
Significance Statement
}

Most eukaryotic organisms contain a distinct group of U12-type introns that are spliced by the minor spliceosome. While U12-type introns comprise only $0.5 \%$ of all introns, the U12 splicing machinery is essential with minor spliceosome mutations linked to developmental defects in both humans and plants. Structural analysis of the activated human minor spliceosome identified four novel splicing factors, including RBM48, as key components without providing direct evidence of function in humans. Here, we provide genetic evidence that human RBM48 is an essential U12-specific splicing factor with similar defects to an RBM48 mutant in maize. Our data points to shared biological processes in plants and animals that depend upon efficient U12 splicing to regulate growth and development. 


\section{Introduction}

Accurate splice site recognition and precise removal of introns during pre-mRNA processing is fundamental for gene expression in eukaryotes ${ }^{1-3}$. The vast majority of introns are spliced by the major spliceosome and are categorized as U2-type (or major) introns ${ }^{4,5}$. A second group of introns are spliced by the minor spliceosome and are categorized as U12-type (or minor) introns ${ }^{6}$. U12-type introns constitute $\sim 0.5 \%$ of all introns in species with a minor spliceosome ${ }^{6}$. In contrast to U2-type, U12-type introns have highly conserved 5' splice site and branch point sequences, tolerate distinct 5' and 3' terminal dinucleotides, and lack 3' polypyrimidine tracts ${ }^{7,8}$. Both U12-type introns and U2-type introns exist side-by-side within Minor Intron-containing Genes (MIGs) ${ }^{6}$. Most of the 758 MIGs identified in humans contain only a single U12-type intron ${ }^{9}$.

Defects in pre-mRNA splicing by cis- or trans-acting mutations have been linked to approximately $60 \%$ of human diseases ${ }^{10-12}$. While many mutations impact splicing of U2-type introns ${ }^{13}$, anomalous splicing of U12-type introns can cause developmental defects in both plants and animals ${ }^{14-19}$. Mutations in minor spliceosome factors impact normal splicing of a subset of MIGs in both humans and maize that result in cell differentiation defects ${ }^{20-22}$. For example, aberrant differentiation and proliferation of myeloid precursors associated with myelodysplastic syndrome (MDS) can be caused by mutations in the spliceosomal gene ZRSR2, which impairs splicing of U12-type introns leading to excess intron retention ${ }^{22}$. MDS patients with ZRSR2 mutations display peripheral cytopenia and accumulate abnormal myeloid cells due to defective hematopoiesis of myeloid precursors in the bone marrow, resulting in an increased risk to acute myeloid leukemia $(\mathrm{AML})^{23}$. In maize, the corresponding ZRSR2 ortholog is disrupted by the hypomorphic rough endosperm3 (rgh3) allele which results in aberrant endosperm differentiation and proliferation as well as impaired splicing and retention of U12type introns ${ }^{21}$. A comparison of MIGs affected in the human ZRSR2 and maize rgh3 mutants identified conserved cellular and molecular pathways associated with cell proliferation and expression of terminal cell fates ${ }^{21}$.

Major and minor spliceosomes are complex molecular machines displaying remarkable similarity ${ }^{6}$. Many of the core and auxiliary protein components are postulated to be shared between the two complexes. To date, only 12 have been identified to be specific to the minor spliceosome $^{6,24}$. One of these U12-specific splicing factors, RNA Binding Motif Protein 48 (RBM48) is co-conserved in eukaryotes with ZRSR2 and shown to be required for efficient splicing of U12-type introns in maize ${ }^{25}$. Maize $r b m 48$ mutants share a stark phenotypic similarity 
with rgh3 mutants in endosperm developmental defects, as both loci exhibit increased cell proliferation and decreased cell differentiation ${ }^{25}$. Maize RBM48 interacts with core U2 Auxiliary Factor (U2AF), Armadillo Repeat-Containing Protein 7 (ARMC7), and RGH3, suggesting the components of both major and minor spliceosomal complexes interact during pre-mRNA processing. A recent structural analysis of a purified human minor spliceosome complex confirmed the interaction of RBM48 with ARMC7. This complex in turn binds to the 5' cap of U6atac, potentially facilitating U12 splicing by stabilizing the intronic sequence in addition to the minor spliceosome itself ${ }^{24}$. However, there is no direct genetic evidence demonstrating a U12 splicing role for RBM48 in human cells.

Herein, we generated a CRISPR/Cas9 mediated functional knockout of RBM48 in human K-562 chronic myeloid leukemia cells. Using comprehensive transcriptome profiling, we demonstrate that RBM48 is required for efficient splicing of U12-type introns indicating conservation of function between plants and humans. Comparative analyses identified a subset of conserved MIGs that are affected in both maize and human RBM48 knockout mutants. These data identify biological processes impacting cell proliferation and development that have maintained common post-transcriptional RNA processing mechanisms since the divergence of plants and animals.

\section{Results}

\section{Targeting human RBM48 for Cas9-mediated cleavage in K-562 cells}

The CRISPR/Cas9 genome editing system was implemented to generate a functional knockout of $R B M 48$ in K-562 cells. Two single-guide RNAs (sgRNA) were designed to target the $\mathrm{N}$-terminal (sgRNA\#1) and C-terminal (sgRNA\#2) regions of the RNA Recognition Motif (RRM) domain within the RBM48 genomic locus. The position, sequence, and approximate cleavage site of each sgRNA is displayed schematically in Figure 1A. To determine the efficacy of Cas9mediated DNA cleavage at each targeting site, genomic DNA (gDNA) was analyzed by the Surveyor nuclease assay from cell populations at harvest following puromycin selection (day 0 ), as well as 10 and 16 days post-selection (Fig. 1B). Insertion-deletion (indel) polymorphisms generated within the RBM48 genomic locus were detected on day 0 targeted cell populations by heteroduplex cleavage fragments of 309 and 272 base pair (bp) for sgRNA\#1- and 332 and 266 bp in sgRNA\#2. No detectable fragments were observed in digested vector control (VC) amplicons. Indels were detected in each targeted population at 10 days post-selection. By day 16, digested fragments were undetectable from sgRNA\#1-targeted DNA. By contrast, cleavage 
fragments from DNA targeted by sgRNA\#2 were stable and appeared to intensify at 16 days post selection.

Growth of the transfected cell populations was monitored over the 16-day post-selection time interval (Fig. 1C and D). Over the first 8 days, cell growth was reduced in all 3 cohorts as a result of the transfection procedure (Fig. 1C). While the VC and sgRNA\#2 cells began to recover at 10 days post-selection, proliferation lagged in the sgRNA\#1-targeted K-562 cell population as compared to VC cells ( $q=0.002)$. By day 16 however, the sgRNA\#1 cells regained vigor with a comparable number of viable cells from both Cas9-targeted and VC populations (Fig. 1D).

The indels generated within the two RBM48-targeted cell populations were further investigated by Sanger sequencing and Tracking of Indels by DEcomposition (TIDE) analysis on day 16 post-selection cells. Sequencing chromatograms flanking the protospacer adjacent motif (PAM) site of sgRNA\#1 (Fig. 1E; upper panel) and subsequent TIDE analysis (Fig. 1F; left), showed that the sgRNA\#1-targeted cells were a nearly homogenous population predominantly harboring wild-type RBM48 by day 16 post-selection. By contrast, multiple sequence trace peaks immediately following the sgRNA\#2 Cas9 cleavage site were observed (Fig. 1E; lower panel). The frequency of wild-type RBM48 sequences within the heterogeneous sgRNA\#2 cell population by TIDE analysis was only $19.8 \%$, while the total Cas 9 cleavage efficiency was approximately $72 \%$ with nearly $30 \%$ comprising a 1 bp insertion (Fig. $1 \mathrm{~F}$; right). From these observations, we conclude that sgRNA\#1 was inefficient in generating stable targeted-indels, which led to the proliferation of unmodified RBM48 wild-type cells as the dominant population. However, the sgRNA\#2 targeting site was highly efficient at generating stable indels without apparent growth defects, enabling us to readily propagate this cell population. Therefore, the VC and sgRNA\#2-targeted cell populations were established and maintained as independent K-562 cell sublines, hereafter referred as VC and $R B M 48 \mathrm{KO}$, respectively.

\section{Loss of full length RBM48 in the RBM48 KO subline}

Loss of $r b m 48$ in maize impairs splicing of U12-type introns ${ }^{25}$. To elucidate whether human RBM48 has a conserved role in U12-dependent splicing, we performed mRNA-seq analysis on six unique passages of both $R B M 48 \mathrm{KO}$ and corresponding $\mathrm{VC}$ populations. Prior to analyzing the mRNA-seq data for splicing defects, $R B M 48$ expression was compared between the heterogeneous $\mathrm{KO}$ and $\mathrm{VC}$ populations to determine the impact of CRISPR/Cas9 mutagenesis. In agreement with the sgRNA\#2 TIDE analysis in Figure 1F, a disparity in 
summed read depth was clearly visible within the targeting region of $R B M 48$ exon 3 in the KO population in comparison to VC (Fig. 2A). However, RT-qPCR assays for expression of all three RBM48 transcripts (Fig. 2B) showed no significant differences between the $\mathrm{KO}$ and VC populations over six passages indicating that Cas9-targeting did not affect overall transcription of the RBM48 gene. Through further analysis, 12 indel-harboring transcript sequences were identified that were maintained within all six passages of the RBM48 $\mathrm{KO}$ subline. Figure $2 \mathrm{C}$ displays the indel positions and their predicted translational consequences in relation to NM_032120.4 and NP_115496.2, respectively. Within the heterogeneous KO subline, we found evidence for two mutant transcripts resulting in loss of a single amino acid and ten mutant transcripts resulting in frameshifts and truncation of the C-terminal region of the RBM48 protein product in all three coding variants.

To test whether sgRNA\#2 targeting impacts wild-type RBM48 protein levels, we generated a third K-562 cell subline that carried a Cas9-mediated C-terminal Myc epitope tag as a tool to monitor protein truncation or loss of expression by sgRNA\#2 targeting as described in Figure S1. The RBM48 C-terminal region was selected to avoid a potential synthetic interaction due to overlap between the regulatory regions of $R B M 48$ and the adjacent PEX1 gene. CRISPR/Cas9 targeting in the RBM48-Myc subline using sgRNA\#2 (KO) resulted in indel generation similar to, but with lower efficiency than, that observed in the original $R B M 48 \mathrm{KO}$ subline (Fig. 3A and B). Western blot analyses of three independent passages of the RBM48Myc VC and $\mathrm{KO}$ cells revealed a significant reduction in RBM48-Myc protein levels in the sgRNA\#2-targeted KO cell populations compared to VC (Fig. 3C; $p=0.0051$ ). We concluded that $R B M 48 \mathrm{KO}$ cells had drastically reduced expression of full length RBM48 protein and that this subline could be used to determine the effects of the rbm48 mutation on transcript processing.

\section{Disruption of U12-dependent splicing in $R B M 48 \mathrm{KO}$ cells}

To assess whether the RBM48 RNA processing role is conserved in maize and humans, transcriptome profiles of the RBM48 KO and VC populations were analyzed for intron splicing defects. Intronic and flanking exon-exon junction read counts for each individual U2- and U12type intron expressed in the K-562 cell sublines were assessed for significance using Fisher's Exact Test (Dataset S1). A minimum sum of 10 total exon-exon junction and intron reads with an intron read density $>0$ in both $\mathrm{VC}$ and $\mathrm{KO}$ populations were required to test an intron. Of the 668 U12-type introns tested, 278 (42\%) were significantly retained in the RBM48 KO population in comparison to VC $(q \leq 0.05)$. Gene Ontology $(\mathrm{GO})$ term enrichment analysis identified cellular 
localization as the most significantly enriched term $(q \leq 0.05)$ among MIGs with U12-type intron retention (Table S1).

Four affected U12-type introns in the DIAPH1, MAPK1, MAPK3, and TXNRD2 genes were selected for validation by RT-qPCR. Based on our mRNA-seq data, each of these genes exhibited significantly increased read depth $(q \leq 0.05)$ in the U12-type intronic regions in RBM48 KO populations compared to VC (Fig. 4). We designed primers to detect both U12-type intron retention and total transcript levels by RT-qPCR for each of the selected MIGs (Fig. 4). No significant differences were found in the total transcript expression levels between the $R B M 48$ $\mathrm{KO}$ and VC populations. By contrast, expression levels of all four U12-type introns were significantly increased, suggesting intron retention in these transcripts. These findings indicate that in humans, RBM48 plays a role in post-transcriptional RNA processing and U12-dependent splicing, but that U12-type intron retention does not necessarily reduce the transcript levels of all genes where the U12-type introns are retained.

Maize rbm48 mutants cause specific retention of U12-type introns with impacts on U2type introns that cannot be distinguished from random noise ${ }^{25}$. The $\log _{2}$ fold-change $\left(\log _{2} \mathrm{FC}\right)$ distributions between human RBM48 KO and VC for all U2-type and U12-type introns are plotted in Figure 5A. Similar to defects observed in maize rbm48 mutants ${ }^{25}$, U12-type introns are shifted to greater overall $\log _{2} \mathrm{FC}$ values than U2-type introns. In contrast to the $42 \%$ of U12type introns, only $9 \%$ of U2-type introns are significantly retained in $R B M 48 \mathrm{KO}$ (Fig. $5 \mathrm{~B}$ ). This indicates that like maize, loss of RBM48 in humans specifically increases retention or missplicing of U12-type intron sequences.

\section{Conservation of mis-spliced genes shared between U12 splicing mutants of human and maize}

The aberrant splicing of multiple U12-type introns poses a challenge to unambiguously identify target genes that cause defective phenotypes. We performed comparative analysis and searched for MIGs that display orthologous defects of U12-type intron splicing between the RBM48 mutants of human and maize. Gault et al. found 36 human MIGs that share homology with 57 maize $\mathrm{MIGs}^{21}$. These candidate orthologs have conserved domain structure and high sequence similarity of greater than 80 -bit score using reciprocal blastp. The imbalance of human to maize MIGs is a result of differential gene duplication and divergent retention of duplicated genes since the split of animal and plant lineages ${ }^{26}$. Therefore, several single copy human MIGs share homology with two or more gene copies in maize. Of these 36 human MIGs, 
$58 \%$ are significantly mis-spliced in RBM48 KO cells. As shown in Figure 5C, 18 human MIGs share mis-splicing with 22 homologous maize MIGs in rbm48 endosperm (Table 1). To validate these RNA-seq comparisons, we selected four genes, SMYD2, TAPT1, WDR91 and ZPR1, that showed significant U12-type intron retention in both human $R B M 48 \mathrm{KO}$ and maize rbm48 mutants (Fig. 6). As shown in Figure 6A, the RBM48 KO cells exhibited increased RNA-seq read depth in U12-type intronic regions compared to VC. When analyzed by RT-PCR, each of these genes also display stronger band intensity of the U12-type intron retained transcript in RBM48 KO mutants (Fig. 6B). Moreover, corresponding RT-PCR assays of maize homologs show increased U12-type intron retention products in maize rbm48 mutants relative to wild-type (Fig. 6B).

A similar example of U12-type intron retention in conserved MIGs has been observed between human ZRSR2 and maize rgh3 mutant samples ${ }^{21,22}$. Previously, we reported strong overlap of U12-type intron targets between maize rbm48 and rgh3 mutants as well ${ }^{25}$. Interestingly, of the 36 human and 57 maize homologous MIGs, 9 human MIGs are significantly mis-spliced in our RBM48 KO and the ZRSR2 mutants ${ }^{22}$ corresponding to 11 significantly misspliced homologous MIGs in the maize $r b m 48^{25}$ and $r g h 3^{21}$ mutants (Table 1 ).

\section{Discussion}

Components of the minor spliceosome play important roles in promoting normal multicellular processes in both plants and animals. However, the mechanisms by which they impact cell development and differentiation are not well understood. Mutations in minor spliceosomal factors cause pleiotropic molecular defects affecting a large proportion of MIGs ${ }^{15}$, 17, 21, 27-29. This has made it difficult to delineate the causative genes underlying the pleiotropic phenotypes of MIG spliceosomal factor mutants, even if they exhibit highly similar characteristics.

Recently, cryo-electron microscopic analysis of purified human minor spliceosome structure revealed RBM48 and ARMC7 bind to the 5' cap of U6atac ${ }^{24}$. In this context, we previously demonstrated the protein-protein interaction of RBM48 with ARMC7 is evolutionary conserved between maize and humans ${ }^{25,30}$. Based on the role of maize RBM48 in U12-type intron splicing, we hypothesized human RBM48 would also impact U12-type intron splicing. We investigated RBM48 function by generating a CRISPR/Cas9 knockout in the human K-562 cell line and found that this role is conserved between humans and maize. Approximately $42 \%$ of U12-type introns analyzed in the RBM48 KO subline were significantly retained, with their corresponding MIGs associated with several different pathways that are essential to growth and 
differentiation. We further determined that there are 18 orthologous MIGs mis-spliced in both human and maize RBM48 mutants. These observations identified a small group of MIGs as candidates for potential trans-species drivers of mutant phenotypes.

With the exception of FRA10AC1, mutations that either alter or abolish gene expression of these 18 conserved MIGs have been documented in the literature. In each case, the MIG mutations result in phenotypes displaying a broad spectrum of developmental defects in either humans or plants, several of which are reported in both species. For example, mutations in human ALG12 lead to Congenital Disorders of Glycosylation type Ig (CDG-lg). CDG-lg patients display a wide range of pleiotropic effects, including but not limited to hypotonia, developmental delay, and abnormal blood clotting ${ }^{31-33}$. A mutation in the homologous Arabidopsis ALG12 gene disrupts ER-mediated degradation of brassinolide receptors conferring a dwarf mutant phenotype to the plant ${ }^{34}$. Similarly, mutations in the chromatid cohesin SMC3 and homologous $T T N$ genes lead to pleotropic developmental defects in both humans and plants, respectively ${ }^{35}$, 36 .

Mutations in U12 splicing factors are also reported to inhibit cell differentiation and promote cell proliferation in both plants and animals. Somatic mutations of human ZRSR2 cause suppression of myeloid cell differentiation and increased proliferation of myeloid progenitor cells $\mathrm{s}^{22}$. Similarly, maize mutants in both $\mathrm{rgh} 3$ and $\mathrm{rbm} 48$ suppress endosperm differentiation and increase proliferation of endosperm in tissue culture ${ }^{21,25}$. Within several of the conserved MIGs identified herein, mutations have been linked to abnormal cell proliferation or cancer in humans. For example, mutations in $B R C C 3$, which encodes a cell cycle regulator involved in G2/M phase transition, have been linked to myelodysplastic syndrome (MDS) as well as breast and cervical cancers ${ }^{37-39}$. Mutations in E2F3 have been associated with retinoblastoma, bladder and lung cancer ${ }^{40-42}$.

Despite these observed similarities, cell type- and lineage-specific differences play a large role in phenotypic effects of U12-type intron retention. Differential effects on in vitro cell viability are observed with targeted loss of RBM48 in genome-wide CRISPR/Cas 9 dropout screens where RBM48 is deemed essential in some human cell lines and dispensable in others $^{30,43-47}$. In a recent meta-analysis of 17 CRISPR screens conducted using three different large-scale libraries, $R B M 48$ is found to be essential for survival in 4 out of 7 leukemia-derived cell lines $^{44}$. This includes the OCI-AML3, OCI-AML2, HL-60 and HAP1 lines. However, viability is unaffected in MV4-11, KBM-7 and $\mathrm{K}-562$ cells deficient in RBM48, which agrees with our results in the $R B M 48 \mathrm{KO} \mathrm{K}-562$ subline. Interestingly, the genetic functions of ZRSR2 or RBM48 
are highly overlapping in these 7 hematopoietic cell lines with ZRSR2 and RBM48 being essential in OCI-AML3, OCI-AML2, and HL-60, while both genes are dispensable in K-562, KBM-7, and MV4-11 44 . These 7 cell lines each retain a degree of lineage plasticity and can be induced to further differentiate in response to defined stimuli. For example, the adherent HAP1 cell line was isolated from an attempt to generate induced pluripotent stem cells from KBM-7 suspension cells and as a result, no longer express the hematopoietic markers CD43 and $\mathrm{CD}_{4} 5^{48}$. These two nearly haploid lines also vary in their requirement of $R B M 48$ and $Z R S R 2$ for survival. These cell-type specific differences suggest that phenotypic effects of U12-type intron retention are likely dependent on the lineage-specific differentiation potential of each cell line with disruption of MIG-specific pathways resulting in lethality in some cell lines but not others.

The recent purified structure of the human minor spliceosome revealed six conserved amino acid residues of RBM48 that mediate the interaction of RBM48-ARMC7 protein complex with $U 6 \mathrm{atac}^{24}$. Of these, four amino acid residues are N-terminal to the conserved RRM domain of RBM48. Thus, the position of the sgRNA\#1 is predicted to interrupt three of these residues, possibly negating a vital RBM48-U6atac interaction and rendering a lethal or anti-proliferative phenotype to the resultant host cells. In contrast, the position of sgRNA\#2 spanning the distal region of the RRM domain does not impact the expression of these conserved residues, allowing targeted, mutant K-562 cell sublines to proliferate sufficiently for RBM48 KO analysis. In vertebrates, RBM48 and ARMC7 are differentially compartmentalized in the nucleus and cytosol, respectively ${ }^{49}$ (Human Protein Atlas available from http://www.proteinatlas.org), which warrants investigation of in vivo significance of RBM48-ARMC7 interactions.

A combination of in vivo co-expression, Bimolecular Fluorescence Complementation (BiFC), and in vitro pull-down assays showed that maize RBM48 interacts with RGH3 and the core U2 splicing factors U2AF1 and U2AF2 ${ }^{24}$. U2AF2 binds to the polypyrimidine tract of U2type introns; whereas U2AF1 makes contact with the acceptor site ${ }^{50}$. Human ZRSR2 binds to the 3' splice site of U12-type introns and is required for the formation of pre-spliceosomal complexes, but ZRSR2 also interacts with U2AF2 and is essential for the second transesterification reaction of U2 splicing ${ }^{6}$. These protein-protein interaction and in vitro splicing observations suggest that the major and minor spliceosomes interact for splice site selection. However, the splicing phenotypes for mutants in human ZRSR2 as well as maize rgh3 argue that in vivo protein functions are specific for U12-type introns ${ }^{21,22,25}$. The precise role of RBM48ZRSR2 interactions in U12 splicing mechanisms remains to be determined. Perhaps, interactions of RBM48 with ZRSR2 may promote formation of the activated minor spliceosome. 
Developmental gene expression is intricately regulated in a temporal, cellular, and tissue-specific manner beginning in the early stages of embryogenesis. Surprisingly, the inefficient splicing of U12-type introns often perturbs the coding potential but not the expression level of MIGs, many of which are essential for development. We found no changes in total transcript expression levels in our validation assays and similar observations have been made for maize rgh3 and $r b m 48^{21,25}$. Consequently, the mutant phenotypes observed for U12dependent splicing defects are likely a direct result of the disrupted post-transcriptional processing of MIG transcripts. Further elucidation of the mechanism(s) by which retention of U12-type introns result in mutant phenotypes will not only entail biochemical and genetic analysis of individual MIGs but will also require comprehensive profiling of their expression in diverse cells and tissues at different stages of development, both at the levels of transcription and translation.

\section{Materials and Methods}

\section{RBM48-Targeting CRISPR/Cas9 Constructs and Donor Templates}

Vector and target-specific guide RNA (sgRNA) were designed as described ${ }^{51}$. The three RBM48-specific sgRNAs (Table S2) were separately cloned into the $\mathrm{pX} 459$ expression vector [pSpCas9(BB)- 2A-Puro; Addgene plasmid ID: 48139] ${ }^{52}$. The single stranded DNA oligonucleotide for homology directed repair to generate a C-terminal RBM48 Myc-epitope tag was obtained from Integrated DNA Technologies (IDT) (Table S2, Fig. S1).

\section{Transfection of K-562 Cells}

K-562 cells (American Type Culture Collection (ATCC) CCL-243) were cultured in Iscove's Modified Dulbecco's Medium (IMDM) (Hyclone), supplemented with $10 \%(\mathrm{v} / \mathrm{v})$ fetal bovine serum (FBS) (Hyclone) and incubated with $5 \% \mathrm{CO}_{2}$ at $37^{\circ} \mathrm{C}$. K-562 cells were seeded in triplicate into 6 -well plates at a density of $2.4 \times 10^{5}$ cells per well in $3 \mathrm{~mL}$ culture media for $24 \mathrm{~h}$ prior to transfection. For RBM48 KO cells, each well was transfected with $0.5 \mu \mathrm{g} \mathrm{pX459}$ construct expressing sgRNA\#1 or sgRNA\#2. VC cells were transfected with $0.5 \mu \mathrm{g}$ pX459 alone. For RBM48-Myc-epitope tagging, cells were co-transfected with $0.5 \mu \mathrm{g}$ X459 construct with or without sgRNA TAG and $0.5 \mu \mathrm{g}$ homology directed repair donor template. Vector DNA was mixed with $0.75 \mu \mathrm{L}$ lipofectamine 3000 and diluted in Opti-Mem I media (Gibco/Thermo Fisher Scientific). Transfected cells were incubated for $24 \mathrm{~h}$ and then selected for $48 \mathrm{~h}$ using 3 $\mu \mathrm{g} / \mathrm{mL}$ puromycin dihydrochloride. Transfectants were resuspended in fresh media and aliquots 
were isolated for cell viability assays and genotyping (day 0). Six-well culture plates were initially seeded with approximately $10^{5}$ cells per well in $3 \mathrm{~mL}$ culture media. Cell viability was monitored every $48 \mathrm{~h}$ for 16 days using trypan blue exclusion assay and counted by hemocytometer. Significant differences were determined with unpaired Student's $t$-tests adjusted for multiple comparisons using the Benjamini and Hochberg False Discovery Rate. Viable cells from day 16 cultures of VC and sgRNA\#2-targeted RBM48 KO K-562 cells were subsequently cultured across six serial passages. Cells from each passage were collected and used for analyses representing six biological replicates of each subline. For the RBM48-Myc tagged cotransfectants, single-cell colonies isolated by serial dilution were expanded for two weeks prior to genotypic analysis. A single homogenous RBM48-Myc cell population was selected and similarly re-transfected with either pX459 or pX459 construct containing sgRNA\#2. Following puromycin selection, cells from three serial passages of VC and heterogeneous sgRNA\#2targeted RBM48-Myc cultures were collected for analyses.

\section{Genomic DNA Extraction and Sanger Sequencing}

Genomic DNA (gDNA) from harvested cell populations was extracted using the E.Z.N.A. Tissue DNA kit (Omega Bio-tek). DNA was amplified using RBM48 specific primer pairs and amplicons were bidirectionally sequenced with the same or custom inner forward and reverse primers (Table S3). Sanger sequencing was performed by Genewiz. The sequencing chromatograms were analyzed visually and the frequency of indels generated at CRISPR target sites were quantified using Tracking Indels by Decomposition (TIDE) analysis ${ }^{53}$.

\section{Surveyor Nuclease Assay}

The genomic region spanning the CRISPR target sites were PCR amplified using primers listed in Table S3. PCR products were hybridized to promote heteroduplex formation with the temperature cycle: $95^{\circ} \mathrm{C}$ for $10 \mathrm{~min} ; 95^{\circ} \mathrm{C}$ to $85^{\circ} \mathrm{C}$ ramping at $-2^{\circ} \mathrm{C} / \mathrm{s} ; 85^{\circ} \mathrm{C}$ to $25^{\circ} \mathrm{C}$ at $0.3^{\circ} \mathrm{C} / \mathrm{s} ; 25^{\circ} \mathrm{C}$ for $1 \mathrm{~min}$ and $4^{\circ} \mathrm{C}$ hold. The resultant DNA products were subjected to SURVEYOR nuclease analysis following instructions provided by the manufacturer (IDT).

\section{RNA Extraction, cDNA Synthesis and RT-PCR}

VC and RBM48 KO cells from the six serial passages were pelleted and subsequently homogenized in TRIzol reagent followed by phase separation. Total RNA was extracted from the supernatants using the RNeasy Plus Universal Kit (Qiagen) per the manufacturer's protocol 
with the inclusion of gDNA digestion using the RNase-Free DNase Set. gDNA-free total RNA was eluted in $30 \mu \mathrm{L}$ RNase free water and the RNA concentration and purity was determined by Nanodrop 2000C. RNA integrity was confirmed by gel electrophoresis using $1 \%$ agarose with ethidium bromide. gDNA-free total RNA $(1 \mu \mathrm{g})$ was reverse transcribed using SuperScript VILO cDNA Synthesis Kit (Life Technologies). Reverse transcription (RT) was performed for 10 min at $25^{\circ} \mathrm{C}, 60 \mathrm{~min}$ at $42^{\circ} \mathrm{C}$, and reactions were terminated by incubation at $85^{\circ} \mathrm{C}$ for $5 \mathrm{~min}$. RT samples were used immediately or stored at $-20^{\circ} \mathrm{C}$. After first strand cDNA synthesis, MIG transcripts were amplified using gene-specific primers as shown in Figure S2 and listed in Table S4.

\section{RT-qPCR}

RT-qPCR was performed in the Bio-Rad CFX96 Real Time System with target genes summarized in Table S5. Reaction mixtures consisted of $1 \mathrm{ng}$ cDNA template, $400 \mathrm{nM}$ specific sense primer, $400 \mathrm{nM}$ specific antisense primer, RNase/DNase-free water, and $1 \mathrm{x}$ SsoAdvanced SYBR Green Supermix (Bio-Rad) in a final volume of $10 \mu \mathrm{L}$. The thermal profile of the PCR followed the SsoAdvanced supermix protocol: initial denaturation at $95^{\circ} \mathrm{C}$ for $30 \mathrm{~s}$ followed by 40 cycles of $95^{\circ} \mathrm{C}$ for $5 \mathrm{~s}$ and $60^{\circ} \mathrm{C}$ for $20 \mathrm{~s}$ with amplification data collected at the end of each cycle. Product specificity was validated with dissociation curves by incubating reactions from 65 to $95^{\circ} \mathrm{C}$ in $0.5^{\circ} \mathrm{C}$ increments for $5 \mathrm{~s}$ each. The Bio-Rad PrimePCR RNA quality, DNA contamination control, and Positive PCR control assays evaluated RNA quality, genomic DNA contamination, and PCR reaction performance respectively. cDNA from each of the six serial passages of the VC and RBM48 KO cell populations were amplified in triplicate for each target gene.

PCR priming efficiencies of U12-type intron retained RNA templates were determined with calibration curves from the $\mathrm{K}-562 \mathrm{RBM} 48 \mathrm{KO}$ subline. cDNA (5 ng/50 $\mu \mathrm{L}$ reaction) was preamplified for 10 cycles with U12-type intron specific primers (Table S5). PCR amplicons were purified and quantified by NanoDrop 2000C. Input DNA copy number was determined using the University of Rhode Island Genomics and Sequencing Center online calculator (https://cels.uri.edu/gsc/cndna.html) with templates ranging from $2 \times 10^{3}$ to $2 \times 10^{6}$ copies/well. Alternatively, a 10-fold dilution series of cDNA from K-562 ATCC CCL-243 cells ranging from 5 pg to $50 \mathrm{ng}$ cDNA/well was assayed. The quantification cycle $(\mathrm{Cq})$ was plotted against the log amount of cDNA input and the relationship between $\mathrm{Cq}$ values and RNA concentration was calculated by linear regression to find a slope and intercept that predicts cDNA amounts and 
correlation coefficient $\left(R^{2}\right)$. Amplification efficiencies $(E)$ were calculated according to the equation $\mathrm{E}=(10$ (-1/slope) -1$) \times 100$ and are expressed as a percentage. The qPCR parameters providing the standard curve for each primer pair are summarized in Table S6. A cDNA positive control inter-run calibrator ( $1 \mathrm{ng} /$ well) was included in every run and the $\Delta$ Cqs were verified to be $<0.01$ between runs. Six reference genes were included and validated with RefFinder ${ }^{54}$. The three genes with the lowest geometric mean rank having a geNorm ${ }^{55}$ stability value $<0.5$ were selected. Gene expression was determined using the Bio-Rad CFX Manager software version 3.0 and calculated using the efficiency corrected model ${ }^{56}$ of the $\Delta \Delta \mathrm{C}_{\mathrm{q}}$ method $^{57}$ modified for normalization by geometric averaging of multiple reference genes ${ }^{55}$. Results are expressed as the ratio of $R B M 48 \mathrm{KO} \triangle \mathrm{Cq}$ expression to $\mathrm{VC} \Delta \mathrm{Cq}$ expression (Relative Normalized Expression). Significant differences were identified with unpaired Student's $t$-tests.

\section{Analysis of RBM48-Myc Protein Expression}

Total protein was extracted from VC and sgRNA\#2-targeted RBM48-Myc cell pellets homogenized in RIPA lysis buffer (50 mM Tris- $\mathrm{HCl}, 150 \mathrm{mM} \mathrm{NaCl}, 1.0 \%$ Triton X-100, 0.25\% sodium deoxycholate, $5.0 \mathrm{mM}$ EDTA) in the presence of $10 \mu \mathrm{L} / \mathrm{mL}$ HALT protease inhibitor cocktail (Thermo Fisher Scientific). Extracts were centrifuged at $15,000 \times \mathrm{g}$ for $15 \mathrm{~min}$ at $4^{\circ} \mathrm{C}$. Supernatant aliquots were frozen in liquid nitrogen and stored at $-80^{\circ} \mathrm{C}$. Total proteins were separated with 7.5\% Mini-PROTEAN TGX sodium dodecyl sulfate polyacrylamide gel (Bio-Rad) with $20 \mu \mathrm{g}$ of protein per lane, quantified via Bradford assay. Protein gels were wet transferred to PVDF membrane (Millipore). Specific proteins were detected using 1:1000 dilution of MycTag (9B11) mouse monoclonal and proliferating cell nuclear antigen (PCNA, D3H8P) XP Rabbit monoclonal primary antibodies (Cell Signaling Technology). Following primary antibody incubation, membranes were washed and incubated with 1:2000 dilution of horseradish peroxidase-conjugated horse anti-mouse IgG (Cell Signaling Technology) and goat anti-rabbit IgG (Santa Cruz Biotechnology) secondary antibodies. Myc-Tag or PCNA signal was detected using SuperSignal West Femto (Thermo Fisher Scientific) or Clarity (Bio-Rad) ECL substrates, and imaged using the Bio-Rad ChemiDoc Touch imaging system. Chemiluminescent signals were quantified with the Image Studio Lite program version 3.1 (LI-COR Biosciences) to normalize the RBM48-Myc protein band density to the PCNA loading control. Statistical analyses were performed using unpaired Student's $t$-tests.

\section{RNA-seq}


Approximately $10^{6}$ cells were collected from each of six serial passages for both VC and RBM48 KO sublines. These 12 biological samples were homogenized in TRIzol and subjected to chloroform/isopropanol extraction per the manufacturer's instructions. Following DNase treatment, the samples were purified using RNeasy MinElute Cleanup Kit (Qiagen). RNA quality was confirmed to have an RNA Integrity Number (RIN) of at least 9.5 with the Agilent Tapestation 2200. Library construction and sequencing were completed at the University of Florida Interdisciplinary Center for Research. The NEBNext Ultra RNA Library Prep Kit for Illumina (New England Biolabs) was used to generate cDNA libraries. The libraries were then pooled and 2 lanes of Illumina HiSeq3000 paired-end 100 bp sequences were generated with a total of $750,648,520$ reads with individual samples ranging 56,844,183 - 75,114,017 reads and averaging 62,554,043 reads.

Raw RNA-seq data were screened to remove adapter sequences using Cutadapt v1.1 $1^{58}$ with the following parameters: --error-rate=0.1 --times $=1$--overlap $=5$--minimum-length $=0$-adapter=GATCGGAAGAGCACACGTCT --quality-base=33. Adapter trimmed sequences were quality filtered/trimmed with Trimmomatic $v 0.22^{59}$ using parameters (HEADCROP:0, LEADING:3, TRAILING:3, SLIDINGWINDOW:4:15, and MINLEN:40) to truncate reads for base quality $<15$ within 4 base windows and kept only reads $\geq 40$ bases after trimming. Only reads remaining in pairs after Trimmomatic were used for subsequent analysis. On average, $28,839,533$ read pairs per sample (range $26,349,516-33,018,146$ ) remained after quality filtering.

Reads were aligned to the human genome sequence assembly (GRCh38) with HiSAT2 ${ }^{60,61}$ using the following parameters: --max-intronlen 100000 -q --pen-noncansplice 6 -no-discordant --rna-strandness RF. Homo_sapiens.GRCh38.87. Annotation (gtf/gff) was used for intron counts and transcripts per million (TPM) normalization. A custom GFF file detailing all non-redundant introns from Homo_sapiens.GRCh38/hg38 was constructed and used to perform U12-type intron retention analysis. Introns within this GFF file were given a unique identifier and annotated as U12- or U2-type introns. Determination of intron type (U12 or U2) was based on a publicly available collection of human U12-type introns ${ }^{9}$ available at https://www.crg.eu/en/programmes-groups/guigo-lab/datasets/u12db-database-orthologousu12-type-spliceosomal-introns. Six-hundred and ninety-five U12-type introns were identified by Alioto $^{9}$ and their sequences and coordinates relative to Human genome annotation version $\mathrm{NCBI35/hg17}$ were available. Conversion of the NCBI35/hg17 iU12-type intron coordinates to Homo_sapiens GRCh38/gh38 was accomplished using a two-step process. The annotation "lift- 
over" utility available from the UCSC genome browser (https://genome.ucsc.edu/cgibin/hgLiftOver) was used to first convert the U12-type intron coordinates from NCBI35/hg17 to CRCh37/hg19, and then again from CRCh37/hg19 to Homo_sapiens.GRCh38.87.

Read counts/gene were determined with the HTSeq-Count utility in the HTSeq package (Ver 0.8.0 $)^{62}$ using the start-stop coordinates of the entire locus and the following parameters: m intersection-nonempty -s reverse). Intron counts were determined with the HTSeq-Count utility (-m intersection-nonempty -s reverse) using the intron GFF file for features. Significant differences in summed intron and exon-exon junction read counts between KO and VC populations were determined by Fisher's Exact Test. Multiple comparison adjustment was performed using the Benjamini-Hochberg False Discovery Rate (FDR) and reported as the adjusted q-value with a significance threshold of 0.05 .

Gene ontology (GO) term enrichment analyses used String-DB with default parameters and the genes having greater than 0 TPM in either VC or KO (genes expressed in either K-562 cell subline) as a customized reference. MIGs analyzed were selected based on significant retention ( $q \leq 0.05$ ) of U12-type introns in RBM48 KO. Non-coding MIGs were excluded. StringDB results were further filtered by enrichment cutoff of $>2.0$ and $q<0.01$. GO term redundancy was filtered with ReviGO using Simrel semantic similarity measurement with allowed similarity $=0.5$.

VC and KO population intron reads and their respective flanking exon-exon junction reads were first filtered for a minimum sum of 10 intronic and exon-exon junction reads in both VC and KO. Read densities were then calculated for intron density (Di) and exon-exon junction density (De) separately as described ${ }^{63}$. Introns were selected for analysis when $\mathrm{Di}>0$ to calculate $\log _{2}$ fold-change of intron splicing efficiency between RBM48 KO and wildtype VC.

\section{Analysis of MIG Homology}

Putative orthologs of maize and human MIGs were identified with reciprocal blastp searches against human and maize protein sequence databases. Human and maize MIGs that returned reciprocal hits with bit scores $>80$ were considered orthologous. We searched for homologous MIGs that share significant intron retention between human RBM48 KO population and maize mutant $r b m 48^{25}$. The position of the intron was determined by direct splice alignment of the protein sequence by SplicePredictor ${ }^{64}$. The position of the intron was considered conserved if the position was within 5 amino acids residues between the two species. 


\section{Acknowledgments}

This work was supported by the National Science Foundation (grant 1412218 to S.L., W.B.B. and A.M.S.); the National Heart Lung and Blood Institute at the National Institutes of Health (grant R01-HL135035 to R.J.W.); the National Cancer Institute at the National Institutes of Health (grant R15-CA182889 to G.J.M.); the Oakland University Research Excellence Fund (to S.L., G.J.M. and R.J.W.) and American Heart Association Innovative Research Grant (to R.J.W.).

\section{References}

1. G. G. Simpson, W. Filipowicz, Splicing of precursors to mRNA in higher plants: mechanism, regulation and sub-nuclear organisation of the spliceosomal machinery. Plant molecular biology 32, 1-41 (1996).

2. Z. J. Lorkovic, D. A. Wieczorek Kirk, M. H. Lambermon, W. Filipowicz, Pre-mRNA splicing in higher plants. Trends in plant science 5, 160-167 (2000).

3. Y. Ru, B. B. Wang, V. Brendel, Spliceosomal proteins in plants. Current topics in microbiology and immunology 326, 1-15 (2008).

4. J. P. Staley, C. Guthrie, Mechanical devices of the spliceosome: motors, clocks, springs, and things. Cell 92, 315-326 (1998).

5. Y. Lee, D. C. Rio, Mechanisms and Regulation of Alternative Pre-mRNA Splicing. Annu Rev Biochem 84, 291-323 (2015).

6. J. J. Turunen, E. H. Niemela, B. Verma, M. J. Frilander, The significant other: splicing by the minor spliceosome. Wiley interdisciplinary reviews. RNA 4, 61-76 (2013).

7. S. L. Hall, R. A. Padgett, Conserved sequences in a class of rare eukaryotic nuclear introns with non-consensus splice sites. J Mol Biol 239, 357-365 (1994).

8. C. L. Will, R. Luhrmann, Splicing of a rare class of introns by the U12-dependent spliceosome. Biol Chem 386, 713-724 (2005).

9. T. S. Alioto, U12DB: a database of orthologous U12-type spliceosomal introns. Nucleic acids research 35, D110-115 (2007).

10. K. H. Lim, L. Ferraris, M. E. Filloux, B. J. Raphael, W. G. Fairbrother, Using positional distribution to identify splicing elements and predict pre-mRNA processing defects in human genes. Proc Natl Acad Sci U S A 108, 11093-11098 (2011).

11. B. Chabot, L. Shkreta, Defective control of pre-messenger RNA splicing in human disease. The Journal of cell biology 212, 13-27 (2016).

12. T. Sterne-Weiler, J. R. Sanford, Exon identity crisis: disease-causing mutations that disrupt the splicing code. Genome Biol 15, 201 (2014).

13. T. A. Cooper, L. Wan, G. Dreyfuss, RNA and disease. Cell 136, 777-793 (2009).

14. P. Edery et al., Association of TALS developmental disorder with defect in minor splicing component U4atac snRNA. Science 332, 240-243 (2011).

15. H. J. Jung, H. Kang, The Arabidopsis U11/U12-65K is an indispensible component of minor spliceosome and plays a crucial role in U12 intron splicing and plant development. Plant J 78, 799810 (2014).

16. W. Y. Kim et al., The Arabidopsis U12-type spliceosomal protein U11/U12-31K is involved in U12 intron splicing via RNA chaperone activity and affects plant development. Plant Cell 22, 3951-3962 (2010).

17. S. Markmiller et al., Minor class splicing shapes the zebrafish transcriptome during development. Proc Natl Acad Sci U S A 111, 3062-3067 (2014).

18. T. Xu, B. M. Kim, K. J. Kwak, H. J. Jung, H. Kang, The Arabidopsis homolog of human minor spliceosomal protein U11-48K plays a crucial role in U12 intron splicing and plant development. Journal of experimental botany 10.1093/jxb/erw158 (2016).

19. $\mathrm{H}$. He et al., Mutations in U4atac snRNA, a component of the minor spliceosome, in the developmental disorder MOPD I. Science 332, 238-240 (2011). 
20. R. Fouquet et al., Maize rough endosperm3 encodes an RNA splicing factor required for endosperm cell differentiation and has a nonautonomous effect on embryo development. Plant Cell 23, 4280-4297 (2011).

21. C. M. Gault et al., Aberrant splicing in maize rough endosperm3 reveals a conserved role for U12 splicing in eukaryotic multicellular development. Proc Natl Acad Sci U S A 114, E2195-E2204 (2017).

22. V. Madan et al., Aberrant splicing of U12-type introns is the hallmark of ZRSR2 mutant myelodysplastic syndrome. Nature communications 6, 6042 (2015).

23. G. C. Shukla, J. Singh, Mutations of RNA splicing factors in hematological malignancies. Cancer Lett 409, 1-8 (2017).

24. R. Bai et al., Structure of the activated human minor spliceosome. Science 10.1126/science.abg0879 (2021).

25. F. Bai et al., RNA Binding Motif Protein 48 Is Required for U12 Splicing and Maize Endosperm Differentiation. Plant Cell 31, 715-733 (2019).

26. F. Wei et al., Physical and genetic structure of the maize genome reflects its complex evolutionary history. PLoS genetics 3, e123 (2007).

27. K. J. Kwak, B. M. Kim, K. Lee, H. Kang, quatre-quart1 is an indispensable U12 intron-containing gene that plays a crucial role in Arabidopsis development. Journal of experimental botany 68 , 2731-2739 (2017).

28. F. Jafarifar, R. C. Dietrich, J. M. Hiznay, R. A. Padgett, Biochemical defects in minor spliceosome function in the developmental disorder MOPD I. RNA 20, 1078-1089 (2014).

29. B. Verma, M. V. Akinyi, A. J. Norppa, M. J. Frilander, Minor spliceosome and disease. Semin Cell Dev Biol 79, 103-112 (2018).

30. T. Hart et al., High-Resolution CRISPR Screens Reveal Fitness Genes and Genotype-Specific Cancer Liabilities. Cell 163, 1515-1526 (2015).

31. C. E. Grubenmann et al., ALG12 mannosyltransferase defect in congenital disorder of glycosylation type lg. Hum Mol Genet 11, 2331-2339 (2002).

32. M. A. Haeuptle, T. Hennet, Congenital disorders of glycosylation: an update on defects affecting the biosynthesis of dolichol-linked oligosaccharides. Hum Mutat 30, 1628-1641 (2009).

33. E. Zdebska et al., Abnormal glycosylation of red cell membrane band 3 in the congenital disorder of glycosylation Ig. Pediatr Res 54, 224-229 (2003).

34. Z. Hong et al., Mutations of an alpha1,6 mannosyltransferase inhibit endoplasmic reticulumassociated degradation of defective brassinosteroid receptors in Arabidopsis. Plant Cell 21, 37923802 (2009).

35. H. Vega et al., Roberts syndrome is caused by mutations in ESCO2, a human homolog of yeast ECO1 that is essential for the establishment of sister chromatid cohesion. Nat Genet 37, 468-470 (2005).

36. C. M. Liu Cm et al., Condensin and cohesin knockouts in Arabidopsis exhibit a titan seed phenotype. Plant J 29, 405-415 (2002).

37. H. E. Boudreau et al., Expression of BRCC3, a novel cell cycle regulated molecule, is associated with increased phospho-ERK and cell proliferation. Int J Mol Med 19, 29-39 (2007).

38. D. Huang et al., BRCC3 mutations in myeloid neoplasms. Haematologica 100, 1051-1057 (2015).

39. F. Zhang, Q. Zhou, Knockdown of BRCC3 exerts an antitumor effect on cervical cancer in vitro. Mol Med Rep 18, 4886-4894 (2018).

40. U. Ziebold, T. Reza, A. Caron, J. A. Lees, E2F3 contributes both to the inappropriate proliferation and to the apoptosis arising in Rb mutant embryos. Genes Dev 15, 386-391 (2001).

41. N. Liu et al., MicroRNA433 reduces cell proliferation and invasion in nonsmall cell lung cancer via directly targeting E2F transcription factor 3. Mol Med Rep 18, 1155-1164 (2018).

42. J. P. Wang, Y. Jiao, C. Y. Wang, Z. B. Xu, B. Zhang, Rb knockdown accelerates bladder cancer progression through E2F3 activation. Int J Oncol 50, 149-160 (2017).

43. A. J. Aguirre et al., Genomic Copy Number Dictates a Gene-Independent Cell Response to CRISPR/Cas9 Targeting. Cancer discovery 6, 914-929 (2016).

44. T. Hart et al., Evaluation and Design of Genome-Wide CRISPR/SpCas9 Knockout Screens. G3 (Bethesda) 7, 2719-2727 (2017). 
45. T. Wang et al., Identification and characterization of essential genes in the human genome. Science 350, 1096-1101 (2015).

46. T. Wang et al., Gene Essentiality Profiling Reveals Gene Networks and Synthetic Lethal Interactions with Oncogenic Ras. Cell 168, 890-903 e815 (2017).

47. K. Tzelepis et al., A CRISPR Dropout Screen Identifies Genetic Vulnerabilities and Therapeutic Targets in Acute Myeloid Leukemia. Cell reports 17, 1193-1205 (2016).

48. J. E. Carette et al., Ebola virus entry requires the cholesterol transporter Niemann-Pick C1. Nature 477, 340-343 (2011).

49. P. J. Thul et al., A subcellular map of the human proteome. Science 356 (2017).

50. J. Valcarcel, R. K. Gaur, R. Singh, M. R. Green, Interaction of U2AF65 RS region with pre-mRNA branch point and promotion of base pairing with U2 snRNA [corrected]. Science 273, 1706-1709 (1996).

51. R. J. Westrick et al., Sensitized mutagenesis screen in Factor V Leiden mice identifies thrombosis suppressor loci. Proceedings of the National Academy of Sciences 114, 9659-9664 (2017).

52. F. A. Ran et al., Genome engineering using the CRISPR-Cas9 system. Nature protocols 8, 22812308 (2013).

53. E. K. Brinkman, T. Chen, M. Amendola, B. van Steensel, Easy quantitative assessment of genome editing by sequence trace decomposition. Nucleic acids research 42, e168 (2014).

54. F. Xie, P. Xiao, D. Chen, L. Xu, B. Zhang, miRDeepFinder: a miRNA analysis tool for deep sequencing of plant small RNAs. Plant molecular biology 10.1007/s11103-012-9885-2 (2012).

55. J. Vandesompele et al., Accurate normalization of real-time quantitative RT-PCR data by geometric averaging of multiple internal control genes. Genome Bio/3, RESEARCH0034 (2002).

56. M. W. Pfaffl, A new mathematical model for relative quantification in real-time RT-PCR. Nucleic acids research 29, e45 (2001).

57. K. J. Livak, T. D. Schmittgen, Analysis of relative gene expression data using real-time quantitative PCR and the 2(-Delta Delta C(T)) Method. Methods (San Diego, Calif.) 25, 402-408 (2001).

58. M. Martin, Cutadapt removes adapter sequences from high-throughput sequencing reads. 201117 , 3 (2011).

59. A. M. Bolger, M. Lohse, B. Usadel, Trimmomatic: a flexible trimmer for Illumina sequence data. Bioinformatics 30, 2114-2120 (2014).

60. M. Pertea, D. Kim, G. M. Pertea, J. T. Leek, S. L. Salzberg, Transcript-level expression analysis of RNA-seq experiments with HISAT, StringTie and Ballgown. Nature protocols 11, 1650-1667 (2016).

61. D. Kim, J. M. Paggi, C. Park, C. Bennett, S. L. Salzberg, Graph-based genome alignment and genotyping with HISAT2 and HISAT-genotype. Nature biotechnology 37, 907-915 (2019).

62. S. Anders, P. T. Pyl, W. Huber, HTSeq--a Python framework to work with high-throughput sequencing data. Bioinformatics 31, 166-169 (2015).

63. Y. Katz, E. T. Wang, E. M. Airoldi, C. B. Burge, Analysis and design of RNA sequencing experiments for identifying isoform regulation. Nature methods 7, 1009-1015 (2010).

64. V. Brendel, L. Xing, W. Zhu, Gene structure prediction from consensus spliced alignment of multiple ESTs matching the same genomic locus. Bioinformatics 20, 1157-1169 (2004). 


\section{Figure Legends}

Figure 1. Functional CRISPR/Cas9-mediated knockout of $R B M 48$ in K-562 cells. (A) Schematic of human RBM48 gene (NM_032120.4) structure displaying the design and position of the sgRNAs used for Cas9 targeting. Gray, green, and blue boxes indicate exons with the exon number indicated below each box. The open reading frame (ORF) is blue, and the RNA Recognition Motif (RRM) is green. Chevron arrowheads indicate position and direction of PCR primers used in Figure 1B, E and F for sgRNA\#1 (red) and \#2 (blue) and are listed in Table S3. The target site of each sgRNA is expanded above the structure. Black, blue, and green text are intronic, ORF and RRM domain sequences, respectively. Black arrowheads mark the predicted cleavage site of Cas9. PAM is the protospacer adjacent motif. (B) Surveyor Nuclease Assay. Heterogeneous K-562 cell populations transfected with VC or sgRNA\#1 (left panel set, red underline) or sgRNA\#2 (right panel set, blue underline), were amplified and digested with Surveyor nuclease to determine indel formation within transfected cell populations at 0,10 and 16 days post-selection. The expected sizes for uncut $(U)$ amplicons spanning the regions targeted by sgRNA\#1 and \#2 were 581 and 599 bp, respectively. C = cut with surveyor nuclease. NTC = no template control. The molecular weight standards are indicated on the left. (C) and (D) Cell viability assays of transfected $\mathrm{K}-562$ cells. Viable cell counts monitored every $48 \mathrm{~h}$ from $72 \mathrm{~h}$ post-puromycin selection (day 0 ) through day 10 are shown in $(C) .{ }^{*} q<0.05$. Viable cell counts on day 16 are shown in (D). (E) Sanger sequencing chromatograms of the amplified region flanking the Cas9 cleavage site targeted by sgRNA\#1 (top panel) and sgRNA\#2 (bottom panel) at day 16 post-selection. (F) TIDE analysis of the chromatograms displayed in Figure 1E. Genomic DNA amplified from VC cell populations was used as non-targeted K-562 input sequence for the analysis.

Figure 2. RBM48 transcript expression from the KO cell population. (A) RNA-seq read depth comparison of RBM48 expression between summed VC and KO libraries. Blue arrowheads mark the position of primers used for RT-qPCR in (B). The sequence of the primers is listed in Table S5. The expanded bottom panel displays the region spanning the sgRNA\#2 target site in exon 3 of the RBM48 sequence. (B) RT-qPCR of relative RBM48 transcript expression between RBM48 KO and VC cells are displayed as the mean \pm SEM from six serial cell passages $(n=6$ per cell subline) and normalized to reference genes HPRT1, IPO8, and PGK1. (C) RBM48 KO expressed transcripts determined from RNAseq. The left panel displays the sequences from the RBM48 sgRNA\#2-targeting region spanning positions 392-467 of the NM_032120.4 transcript variant. The number of nucleotide insertions/deletions (indels) within each sequence are indicated. The predicted translational product of each transcript starting amino acid position 125 of the NP_115496.2 protein accession is shown on the right. Orange text designates changes in protein sequence. Red asterisks mark premature stop codons.

Figure 3. Ablation of RBM48-Myc protein levels by sgRNA\#2-targeting of Cas9. (A) Schematic of RBM48-Myc displaying the sgRNA\#2 targeting site. (B) TIDE analysis of indels present in sgRNA\#2targeted RBM48-Myc cells. (C) Western blot (left panel) and densitometric analysis (right panel) of RBM48-Myc protein levels between KO and VC cell populations. The relative intensities of RBM48-Myc are displayed as the mean \pm SEM from three independent experiments $(n=3)$ and normalized to PCNA levels. ${ }^{*} p<0.001$ by Student's t-test.

Figure 4. RT-qPCR validation of U12-type intron retention in RNA-seq libraries. (A-D) Summed read depth analysis from the six serial passages of $\mathrm{VC}$ and $R B M 48 \mathrm{KO}$ cells are shown at the top of each panel representing the regions of (A) DIAPH1, (B) TXNRD2, (C) MAPK1, and (D) MAPK3 with significant $(q<0.05)$ retention of a U12-type intron in KO cell populations compared to VC. Brace symbol indicates the U12-type intron with increased read depth in KO cells. The bottom panels display RT-qPCR analysis of the relative expression (mean $\pm S E M ; n=6$ per subline) of each respective total transcript (left) and corresponding U12-type intron-containing transcript (right) from $\mathrm{KO}$ samples compared to VC. Expression was normalized to HPRT1, IPO8, and PGK1 expression. Primers for RT-qPCR analysis are available in Table S5. ${ }^{*} p<0.05$. 
Figure 5. Comparative analysis of $\mathrm{U} 12$ intron retention between the RBM48 mutants of human and maize. (A) $\log _{2}$ fold change $\left(\log _{2} F C\right)$ plot of intron expression in KO vs. VC samples. All introns sampled have a minimum sum of 10 intronic and exon-exon junction reads and an intronic read density $>0$ in both $\mathrm{KO}$ and VC samples. (B) Bar graph of the percent of introns significantly retained $(q<0.05)$ in the KO vs. VC sample in U12-type and U2-type introns, respectively. (C) Schematic of overlapping RBM48 splicing defects in homologous human and maize MIGs. Top bar shows total human (purple) and maize (orange) MIGs based on U12DB and ERISdb. The middle bar shows the number of homologous MIGs between humans and maize based on reciprocal blastp analysis. Bottom bar shows homologous MIGs that are significantly mis-spliced in both human and maize RBM48 mutants. Maize data is available from Bai et al. $(2019)^{25}$.

Figure 6. Comparison of U12-type intron retention in homologous MIGs between humans and maize. (A) Display of summed read depth analysis of significantly $(q<0.05)$ increased U12-type intron retention in KO compared to VC cells for SMYD2, TAPT1, WDR91, and ZPR1. The brackets mark the position of the U12-type introns. (B) Semi-quantitative RT-PCR comparison of retention in U12-type introns indicated by band intensity in human (left panel) SMYD2, TAPT1, WDR91, and ZPR1 and their maize (right panel) homologs GRMZM2G457881, GRMZM2G347645, GRMZM2G158179, and GRMZM2G351582, respectively. The position and sequence of the primers used during RT-PCR analysis are displayed in Figure S2 and Table S4, respectively. 
Table 1. Conserved MIGs Mis-spliced in Human and Maize RBM48 Knockout Mutants

\begin{tabular}{|c|c|c|c|c|}
\hline HGNC Symbol & HGNC Gene Name & Human ID $^{\dagger}$ & Maize Homolog & Maize ID $^{\dagger}$ \\
\hline ALG12* & $\begin{array}{l}\text { ALG12 alpha-1,6- } \\
\text { mannosyltransferase }\end{array}$ & Q9BV10 & GRMZM2G152194* & A0A1D6E4A9 \\
\hline BRCC3 & $\begin{array}{l}\text { BRCA1/BRCA2-containing } \\
\text { complex subunit } 3\end{array}$ & P46736 & GRMZM2G096491 & B4FWV0 \\
\hline BTAF1 & $\begin{array}{l}\text { B-TFIID TATA-box binding } \\
\text { protein associated factor } 1\end{array}$ & 014981 & GRMZM2G168096 & A0A1D6MDZ3 \\
\hline DERL2* & derlin 2 & Q9GZP9 & $\begin{array}{l}\text { GRMZM2G117388* } \\
\text { GRMZM2G143817 }\end{array}$ & $\begin{array}{l}\text { Q4G2J6 } \\
\text { Q4G2J5 }\end{array}$ \\
\hline $\mathrm{E}^{2} \mathrm{~F}^{*}{ }^{*}$ & E2F transcription factor 3 & O00716 & $\begin{array}{l}\text { GRMZM2G041701* } \\
\text { GRMZM2G052515* }\end{array}$ & $\begin{array}{l}\text { A0A1D6Q2U1 } \\
\text { A0A1D6ITE9 }\end{array}$ \\
\hline FRA10AC1 & $\begin{array}{l}\text { FRA10A associated CGG } \\
\text { repeat } 1\end{array}$ & Q70Z53 & GRMZM2G001444 & COHFD7 \\
\hline GPN2* $^{*}$ & GPN-loop GTPase 2 & Q9H9Y4 & GRMZM2G093716* & A0A1D6HAG4 \\
\hline IPO9* $^{*}$ & importin 9 & Q96P70 & GRMZM2G457415* & A0A1D6NYK5 \\
\hline MAEA* $^{*}$ & $\begin{array}{c}\text { macrophage erythroblast } \\
\text { attacher }\end{array}$ & Q7L5Y9 & GRMZM2G177026* & B6TF70 \\
\hline SLC66A1 & $\begin{array}{l}\text { solute carrier family } 66 \\
\text { member } 1\end{array}$ & Q6ZP29 & GRMZM2G024733 & COHEC6 \\
\hline SACM1L* & $\begin{array}{c}\text { SAC1 like } \\
\text { phosphatidylinositide } \\
\text { phosphatase }\end{array}$ & Q9NTJ5 & $\begin{array}{l}\text { GRMZM2G047894 } \\
\text { GRMZM2G171080* } \\
\text { GRMZM2G418916* }\end{array}$ & $\begin{array}{c}\text { K7W0E1 } \\
\text { K7UVY0 } \\
\text { A0A1D6HAJ0 }\end{array}$ \\
\hline SMC3 & $\begin{array}{l}\text { structural maintenance of } \\
\text { chromosomes } 3\end{array}$ & Q9UQE7 & GRMZM2G456570 & A0A1D6MEC8 \\
\hline SMYD2* & $\begin{array}{l}\text { SET and MYND domain } \\
\text { containing } 2\end{array}$ & Q9NRG4 & GRMZM2G457881* & $\mathrm{B} 4 \mathrm{FCH} 5$ \\
\hline SMYD3 & $\begin{array}{l}\text { SET and MYND domain } \\
\text { containing } 3\end{array}$ & Q9H7B4 & GRMZM2G080462 & C4IZK2 \\
\hline TAPT1 & $\begin{array}{l}\text { transmembrane anterior } \\
\text { posterior transformation } 1\end{array}$ & Q6NXT6 & GRMZM2G347645 & A0A1D6GJ53 \\
\hline WDR91* & WD repeat domain 91 & A4D1P6 & GRMZM2G158179* & COPKZ7 \\
\hline XRCC5 & $\begin{array}{l}\text { X-ray repair cross } \\
\text { complementing } 5\end{array}$ & P13010 & GRMZM2G137968 & A0A1D6LC75 \\
\hline ZPR1 & ZPR1 zinc finger & 075312 & GRMZM2G351582 & B4FUA0 \\
\hline
\end{tabular}


bioRxiv preprint doi; https://doi.org/10.1101/2020.07.18.209528; this version posted May 13, 2021. The copyright holder for this preprint (which F 19 U $^{\text {as }}$ (not certified by peer review) is the author/funder. All rights reserved. No reuse allowed without permission.

A 3'-...ACGTAAAGAAAGTCCATATATGTTAG $\boldsymbol{\Lambda}$ 'AAACCTTAGAGT...-5,

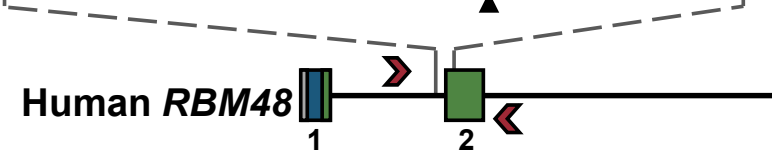

B

Days Post-Selection: 0

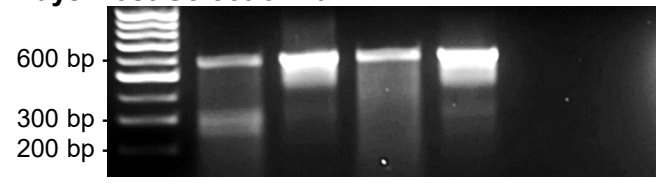

Days Post-Selection: 10

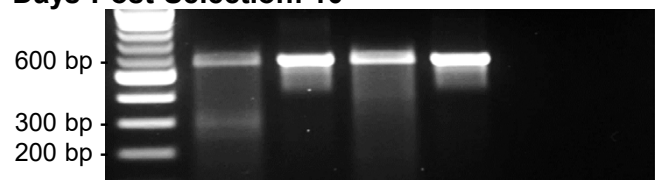

Days Post-Selection: 16

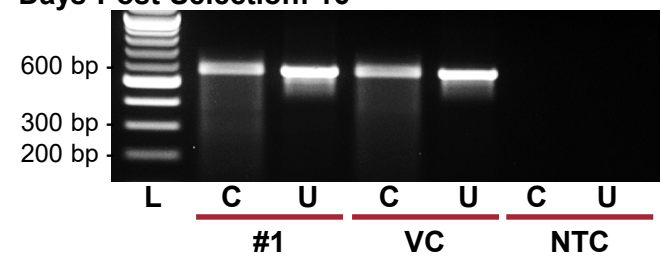

E
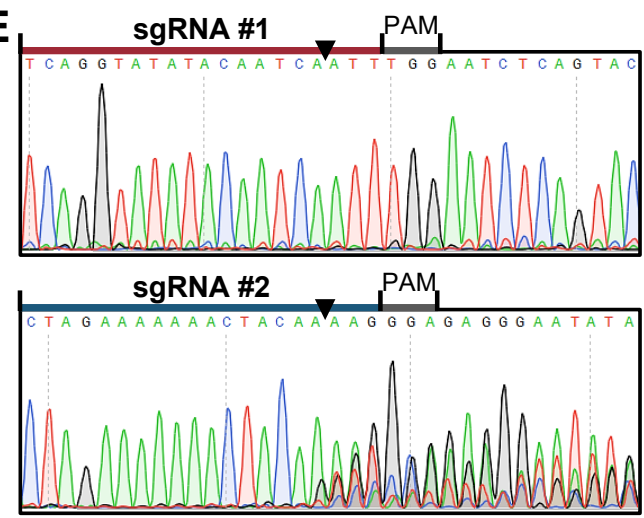

5gRnA \#2

(1) 3'-...CAACTTCTTTGATCTTTTTTTGATGTTTACGCCTTCCGTA...-5',

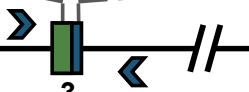

4

C
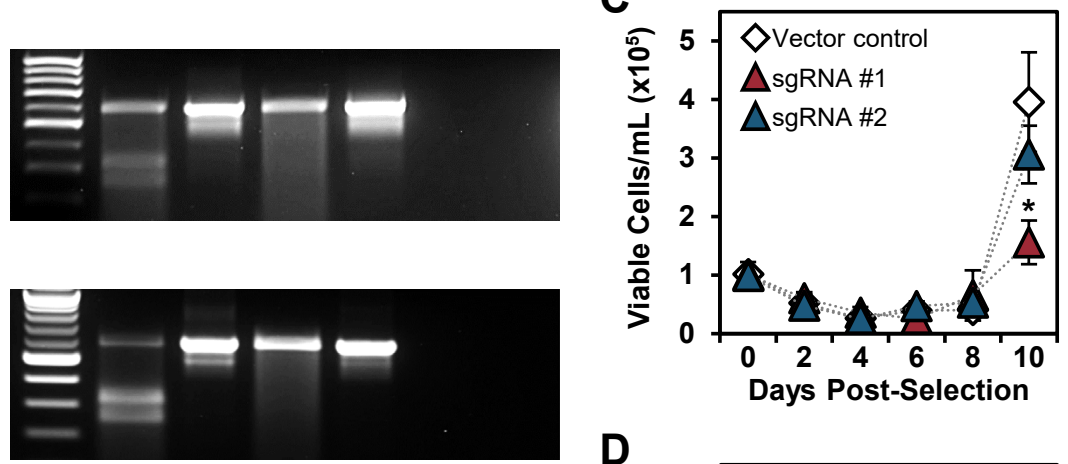

D
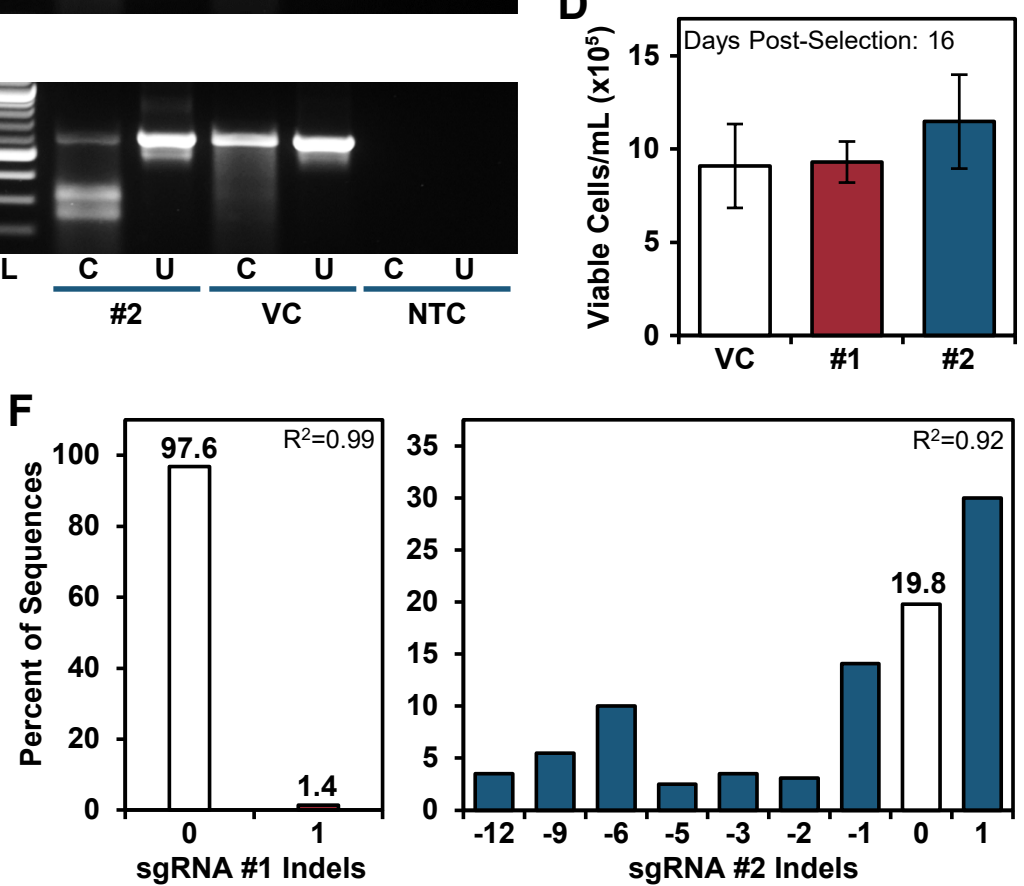


\section{Figure 2}
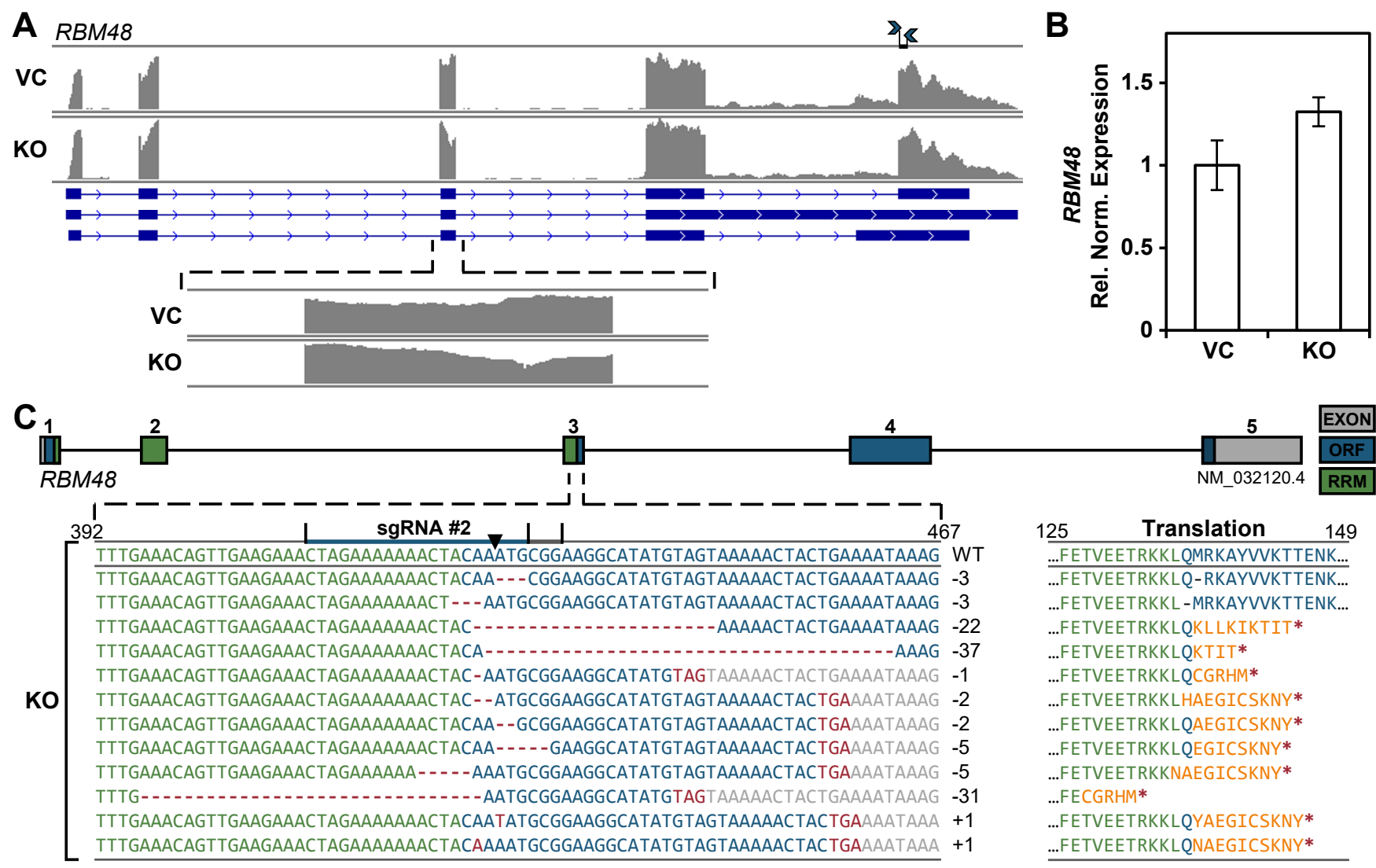

125 Translation 149

...FETVEETRKKLQMRKAYVVKTTENK..

...FETVEETRKKLQ-RKAYVVKTTENK...

...FETVEETRKKL-MRKAYVVKTTENK

...FETVEETRKKLQKLLKIKTIT*

...FETVEETRKKLQKTIT*

...FETVEETRKKLQCGRHM*

...FETVEETRKKLHAEGICSKNY*

....FETVEETRKKLQAEGICSKNY*

...FETVEETRKKLQEGICSKNY*

...FETVEETRKKNAEGICSKNY*

...FECGRHM*

...FETVEETRKKLOYAEGICSKNY* ...FETVEETRKKLQNAEGICSKNY* 
bioRxiv preprint doi: https://doi.org/10.1101/2020.07.18.209528; this version posted May 13, 2021. The copyright holder for this preprint (which F I U revaşot certified by peer review) is the author/funder. All rights reserved. No reuse allowed without permission.
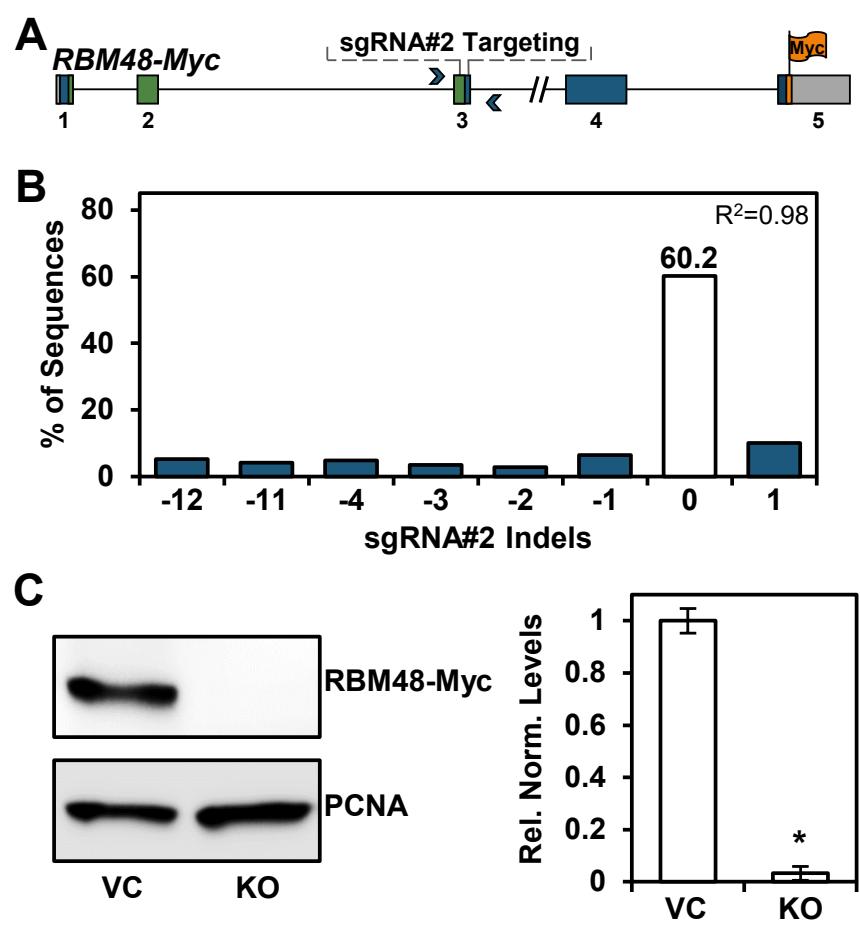
bioRxiv preprint doi:https://doi.org/10.1101/2020.07.18.209528; this version posted May 13, 2021. The copyright holder for this preprint (which

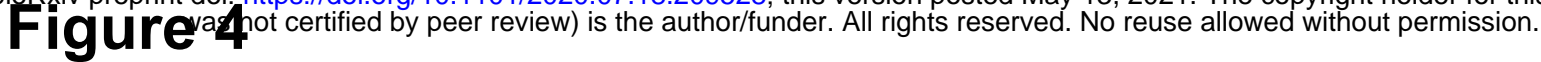
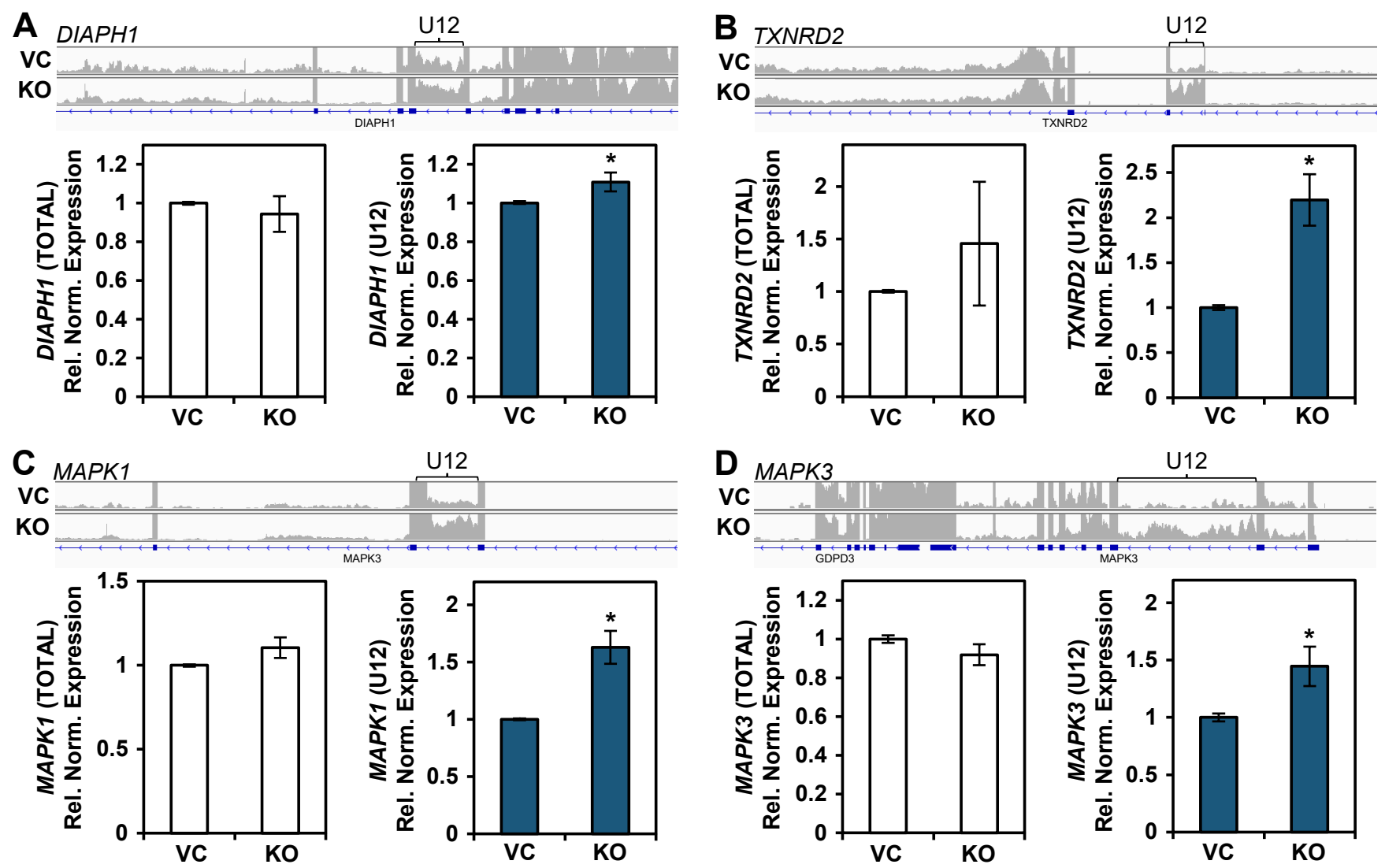
bioRxiv preprint doi: https://doi.org/10.1101/2020.07.18.209528; this version posted May 13, 2021. The copyright holder for this preprint (which F I U revastot certified by peer review) is the author/funder. All rights reserved. No reuse allowed without permission.

A

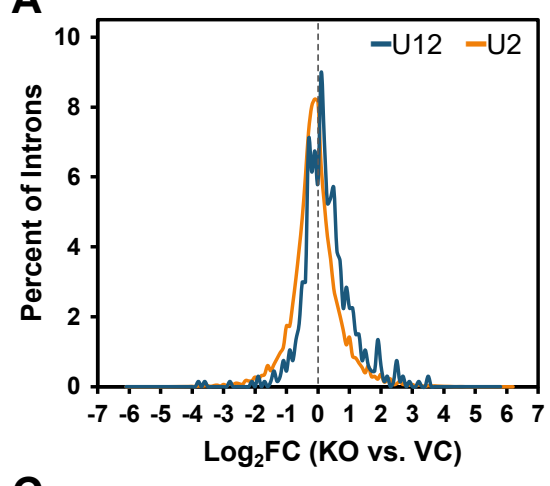

C

Human and Maize MIGs

\section{B}

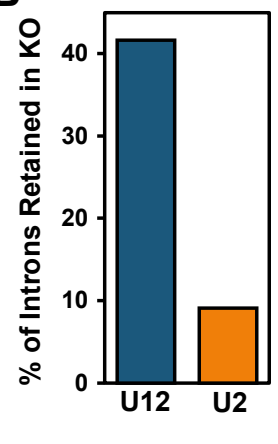

MIG Homologs 36

Human

722

$\prod_{57} \because 351$ Maize

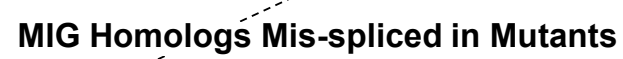

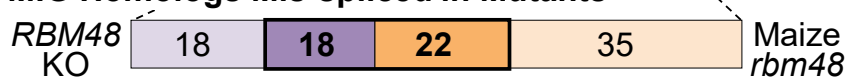



was not certified by peer review) is the author/funder. All rights reserved. No reuse allowed without permission.

Figure 6
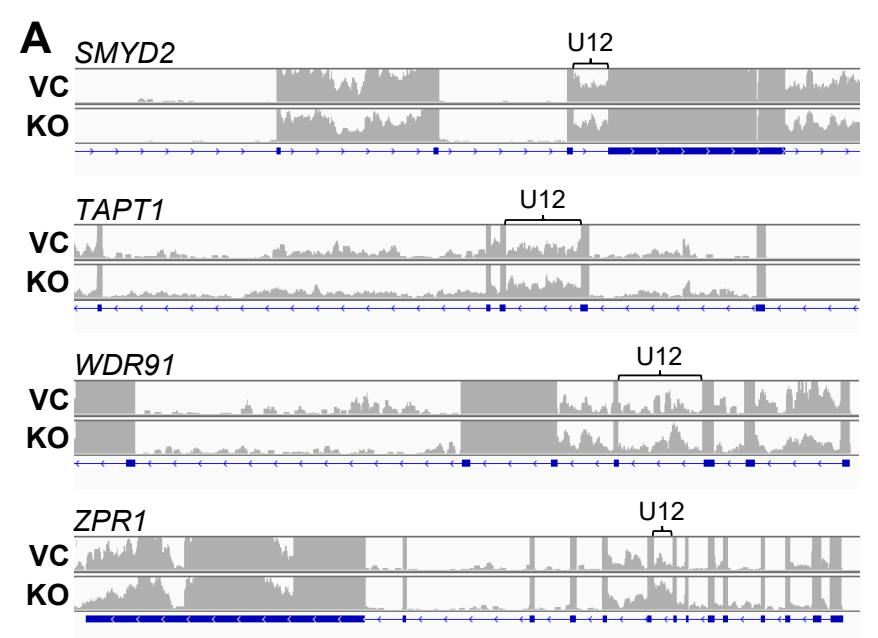

B
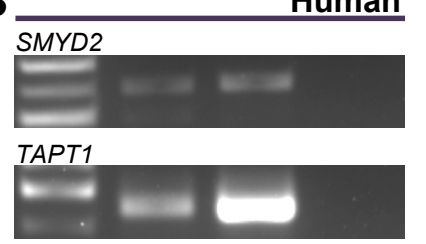

WDR91
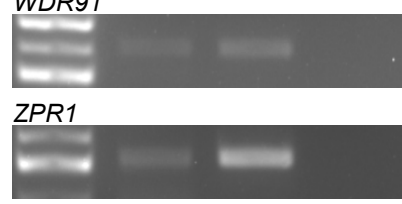

PGK1

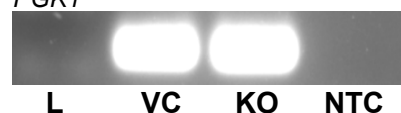

Maize

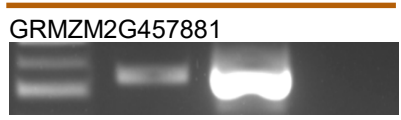

GRMZM2G347645

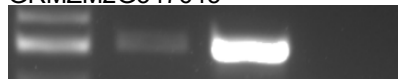

GRMZM2G158179

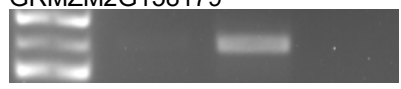

GRMZM2G351582

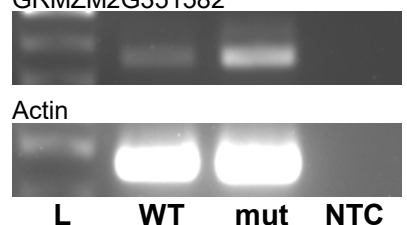

